\title{
Comparative genomics using Fugu reveals insights into regulatory subfunctionalization Adam Woolfe ${ }^{* \dagger}$ and Greg Elgar*
}

\begin{abstract}
Addresses: "School of Biological Sciences, Queen Mary, University of London, Mile End Road, London E1 4NS, UK. ${ }^{\dagger}$ Genomic Functional Analysis Section, National Human Genome Research Institute, National Institutes of Health, Rockville, MD 20870, USA.
\end{abstract}

Correspondence: Adam Woolfe. Email: woolfea@mail.nih.gov

Published: II April 2007

Genome Biology 2007, 8:R53 (doi:10.1 186/gb-2007-8-4-r53)

The electronic version of this article is the complete one and can be found online at http://genomebiology.com/2007/8/4/R53
Received: I December 2006

Revised: 6 March 2007

Accepted: II April 2007

(c) 2007 Woolfe and Elgar; licensee BioMed Central Ltd.

This is an open access article distributed under the terms of the Creative Commons Attribution License (http://creativecommons.org/licenses/by/2.0), which permits unrestricted use, distribution, and reproduction in any medium, provided the original work is properly cited.

\begin{abstract}
Background: A major mechanism for the preservation of gene duplicates in the genome is thought to be mediated via loss or modification of cis-regulatory subfunctions between paralogs following duplication (a process known as regulatory subfunctionalization). Despite a number of gene expression studies that support this mechanism, no comprehensive analysis of regulatory subfunctionalization has been undertaken at the level of the distal cis-regulatory modules involved. We have exploited fish-mammal genomic alignments to identify and compare more than 800 conserved non-coding elements (CNEs) that associate with genes that have undergone fish-specific duplication and retention.

Results: Using the abundance of duplicated genes within the Fugu genome, we selected seven pairs of teleost-specific paralogs involved in early vertebrate development, each containing clusters of CNEs in their vicinity. CNEs present around each Fugu duplicated gene were identified using multiple alignments of orthologous regions between single-copy mammalian orthologs (representing the ancestral locus) and each fish duplicated region in turn. Comparative analysis reveals a pattern of element retention and loss between paralogs indicative of subfunctionalization, the extent of which differs between duplicate pairs. In addition to complete loss of specific regulatory elements, a number of CNEs have been retained in both regions but may be responsible for more subtle levels of subfunctionalization through sequence divergence.
\end{abstract}

Conclusion: Comparative analysis of conserved elements between duplicated genes provides a powerful approach for studying regulatory subfunctionalization at the level of the regulatory elements involved.

\section{Background}

Gene duplication is thought to be a major driving force in evolutionary innovation by providing material from which novel gene functions and expression patterns may arise. Duplicated genes have been shown to be present in all eukaryotic genomes currently sequenced [1] and are thought to arise by tandem, chromosomal or whole genome duplication events. Unless the duplication event is immediately advantageous (for example, by gene dosage increasing evolutionary fitness), the gene pair will exhibit functional redundancy, allowing one 
of the pair to accumulate mutations without affecting key functions. Because deleterious mutations are thought to occur much more commonly than neutral or advantageous ones, the classic model for the evolutionary fate of duplicated genes $[2,3]$ predicts the degeneration of one of the copies to a pseudogene as the most likely outcome (a process known as non-functionalization). Less commonly, a mutation will be advantageous, allowing one of the gene duplicates to evolve a new function (a process known as neo-functionalization). Therefore, the classic model predicts that these two competing outcomes will result in the elimination of most duplicated genes. However, several studies suggest that the proportion of duplicated genes retained in vertebrate genomes is much higher than is predicted by this model [4-6]. This has led to the suggestion of an alternative model whereby complementary degenerative mutations in independent subfunctions of each gene copy permits their preservation in the genome, as both copies of the gene are now required to recapitulate the full range of functions present in the single ancestral gene. This was formalized in the Duplication-Degeneration-Complementation (DDC) model [7] in a process referred to as subfunctionalization.

The key novelty of the DDC model is that, rather than attributing different expression patterns of duplicated genes to the acquisition of novel functions, they are attributed to a partial (complementary) loss of function in each duplicate. In combination they retain the complete function of the pleiotropic original gene, but neither of them alone is sufficient to provide full functionality. For this model to be viable, the subfunctions of the gene are required to be independent so that mutations in one subfunction will not affect the other. The modular nature of many eukaryotic protein-coding sequences as well as cis-regulatory modules (CRMs), such as enhancers or silencers [8], means both can act as subfunctions or components of subfunctions of the gene in subfunctionalization. CRMs are cis-acting DNA sequences, up to several hundred bases in length, thought to be composed of clustered combinatorial binding sites for large numbers of transcription factors that together actuate a regulatory response for one or more genes [9]. The larger number of independently mutable units represented by CRMs, the small size and rapid turnover of transcription factor binding sites, as well as observations that, for many gene duplicates, changes that occur between paralogs are due to changes in expression rather than protein function has led a number of researchers to emphasize that important evolutionary changes might occur primarily at the level of gene regulation [10,11]. Consequently, subfunctionalization is thought most likely to occur by complementary degenerative mutations within regulatory elements.

Teleost fish provide an excellent system to study the DDC model in vertebrates due to the presence of extra gene duplicates that derive from a whole genome duplication event early in the evolution of ray-finned fishes 300-350 million years ago [12-17] This provides the opportunity for comparative analyses of gene duplicates in fish against a single ortholog in tetrapod lineages such as mammals. In particular, for analyses involving important developmentally associated genes, these 'single copies' represent as close as possible the ancestral gene from which the fish duplicates descended, since such genes are often highly conserved in sequence and function throughout vertebrates. We therefore refer to fish-specific duplicate genes as 'co-orthologs' (a term previously used in [18]) as each copy is co-orthologous to the single homolog in tetrapods.

A number of studies on fish duplicated genes have identified cases of subfunctionalization at both the regulatory and protein level. For instance, analysis of the synapsin-Timp genes in the pufferfish Fugu rubripes identified a case of protein subfunctionalization where two isoforms of the SYN gene expressed in human are expressed as two separate genes in Fugu [19]. A number of functional studies on the shared and divergent expression patterns of developmental co-orthologs in fish have also been carried out, for example, eng2 [20], soxy [18] and runx2 [21]. In each case, partitioning of ancestral expression domains for each co-ortholog compared to the single (ancestral representative) gene in mammals was observed via gene expression studies, supporting a process of regulatory subfunctionalization along the lines of the DDC model. Work on identifying the regulatory elements involved has so far been limited to those responsible for divergent expression within the well-studied Hox genes. Santini et al. [22], through comparison to the single tetrapod Hox cluster, identified a number of conserved elements in fish-specific Hox clusters. These appeared to be partitioned between clusters, suggesting they may be responsible for their divergent expression. In addition, the zebrafish hoxb1a and hoxb1b genes, co-orthologs of the $H O X B 1$ gene in mammals and birds, were found to exhibit complementary degeneration of two cis-regulatory elements identified upstream and downstream of the gene, consistent with the DDC model [23]. Similarly, Postlethwait et al. [24] carried out a comparative genomic analysis of the regions surrounding two zebrafish coorthologs, eng2a and eng $2 b$, against the single human ortholog EN2 and found one conserved non-coding element partitioned in each copy, together with a number of elements conserved in both. Both co-orthologs have overlapping expression in the midbrain-hindbrain border and jaw muscles, but eng2a is expressed in the somites and eng $2 b$ is expressed in the anterior hindbrain (both of which are expression domains found in the single mammalian ortholog). Hence, according to the DDC model, they hypothesized that sequences conserved in both co-orthologs represent regulatory elements responsible for overlapping expression domains, whilst conserved sequences specific to each gene are candidates for regulatory elements that drive expression to domains present in the single mammalian ortholog but now partitioned between co-orthologs. Despite these isolated examples, evidence for the DDC model, by way 
of identifying the regulatory elements responsible, remains limited.

Comparison of non-coding genomic sequence across extreme evolutionary distances such as that between fish and mammals to identify regions that remain conserved has proved powerful in identifying sequences likely to be vertebrate-specific distal CRMs (see [25] for a review). Fugu-mammal conserved non-coding elements (CNEs), identified genome-wide, cluster almost exclusively in the vicinity of genes implicated in transcriptional regulation and early development (termed trans-dev genes) with little or no conservation in non-coding sequence outside of these regions; a finding confirmed by a number of recent studies [25-31]. Furthermore, a majority of those CNEs tested in vivo drive expression of a reporter gene in a temporal and spatial specific manner that often overlaps the endogenous expression pattern of the nearby trans-dev gene, confirming this association and their likely role as critical CRMs for these genes [26,29,32-36]. The tight association of CNEs with trans-dev genes is likely the result of the fundamental nature of developmental gene regulatory networks involved in correct spatial-temporal patterning of the vertebrate body plan $[26,37]$.

Fugu-mammal CNEs, enriched for putative CRMs, therefore provide an excellent class of sequences through which to test the DDC model further. In addition, a study has found that at least $6.6 \%$ of the Fugu genome is represented by fish-specific duplicate genes [15], making Fugu an attractive genome in which to identify and analyze regulatory elements involved in subfunctionalization of fish co-orthologs. Transcription factors and genes involved in development and cellular differentiation appear to be overrepresented within duplicated genes in fish genomes [38], improving the chances of identifying suitable candidates. Here, by taking an approach similar to Postlethwait et al. [24], we carried out alignments of genomic sequence around seven pairs of Fugu developmental coorthologs against a number of single mammalian orthologous regions in order to investigate whether differential presence of conserved elements between co-orthologs is consistent with the DDC model of regulatory subfunctionalization.

\section{Results \\ Identification of co-orthologs in the Fugu genome}

Studies into fish-specific duplicated genes have identified a number of examples in the Fugu genome (for example, [15,39]). As with most genes in general, few of these Fugu specific duplicates have CNEs in their vicinity. Suitable gene candidates for study of CNE evolution between teleost-specific gene paralogs were initially identified using 2,330 CNEs derived from a whole-genome comparison of the non-coding portions of the human and Fugu genome [29]. CNE clusters that mapped to the vicinity of a single human genomic region but were derived from two non-contiguous Fugu scaffolds were considered further. We selected seven genomic regions in human that fitted this criterion, each containing clusters of CNEs in the vicinity of a single gene implicated in developmental regulation: BCL11A (transcription factor B-cell lymphoma/leukemia 11A), $E B F 1$ (early B-cell factor 1), FIGN (fidgetin), $P A X 2$ (paired box transcription factor Pax2), SOX1 (HMG box transcription factor Sox1), UNC4.1 (homeobox gene Unc4.1) and ZNF5O3 (zinc-finger gene Znf503). Some of these genes have relatively well characterized roles in early development, such as PAX2 (which plays critical roles in eye, ear, central nervous system and urogenital tract development [40-42], SOX1 (involved in neural and lens development $[43,44], B C L 11 A$ (thought to play important roles in leukaemogenesis and haematopoiesis [45]) and $E B F 1$ (important for B-cell, neuronal and adipocyte development $[46,47]$. FIGN, UNC4.1 and ZNF5O3 are less well characterized, although studies of their orthologs in mouse or rat indicate important roles in retinal, skeletal and neuronal development [48-51].

For each CNE cluster region in the human genome, we identified homologs to the human trans-dev protein on each Fugu scaffold, suggesting the presence of co-orthologous genes. To confirm this, we carried out a phylogeny of these protein sequences together with tetrapod orthologs and all available co-orthologs from the zebrafish genome. In addition, two outgroups utilizing the closest in-paralog as well as an invertebrate ortholog were included in each alignment to help resolve the phylogeny (Figure 1). In all cases where a close paralog could be identified, the Fugu co-ortholog candidates branch with strong bootstrap values with tetrapod orthologs of the target trans-dev gene, rather than the closest paralog, confirming these genes are true co-orthologs. Furthermore, for all phylogenies, the Fugu and zebrafish/medaka sequences branch together after the split with tetrapods, confirming they derive from a fish-specific duplication event. In only one out of three cases (pax2) where two co-orthologous proteins could also be identified in zebrafish does each Fugu copy branch directly with each zebrafish copy, indicating their proteins have followed similar evolutionary paths (Figure 1d). In contrast, the other two cases (sox1 and unc4.1) exhibit a different topology in that both zebrafish coorthologs are more similar to one of the Fugu co-orthologs than the other (although weak bootstrap values for the fish unc4.1 may suggest alternative phylogenies). This is most likely due to species-specific asymmetrical rates of evolution seen between many genes in teleost fish [52], as well as elevated rates of evolution in duplicated genes in general, and pufferfish in particular [38], which may have obscured the true phylogenies in these cases. The given names of the Fugu co-orthologs used in this study (see Materials and methods for more details on nomenclature), their location in the Fugu genome and protein sequence accession codes can be found in Table 1. 


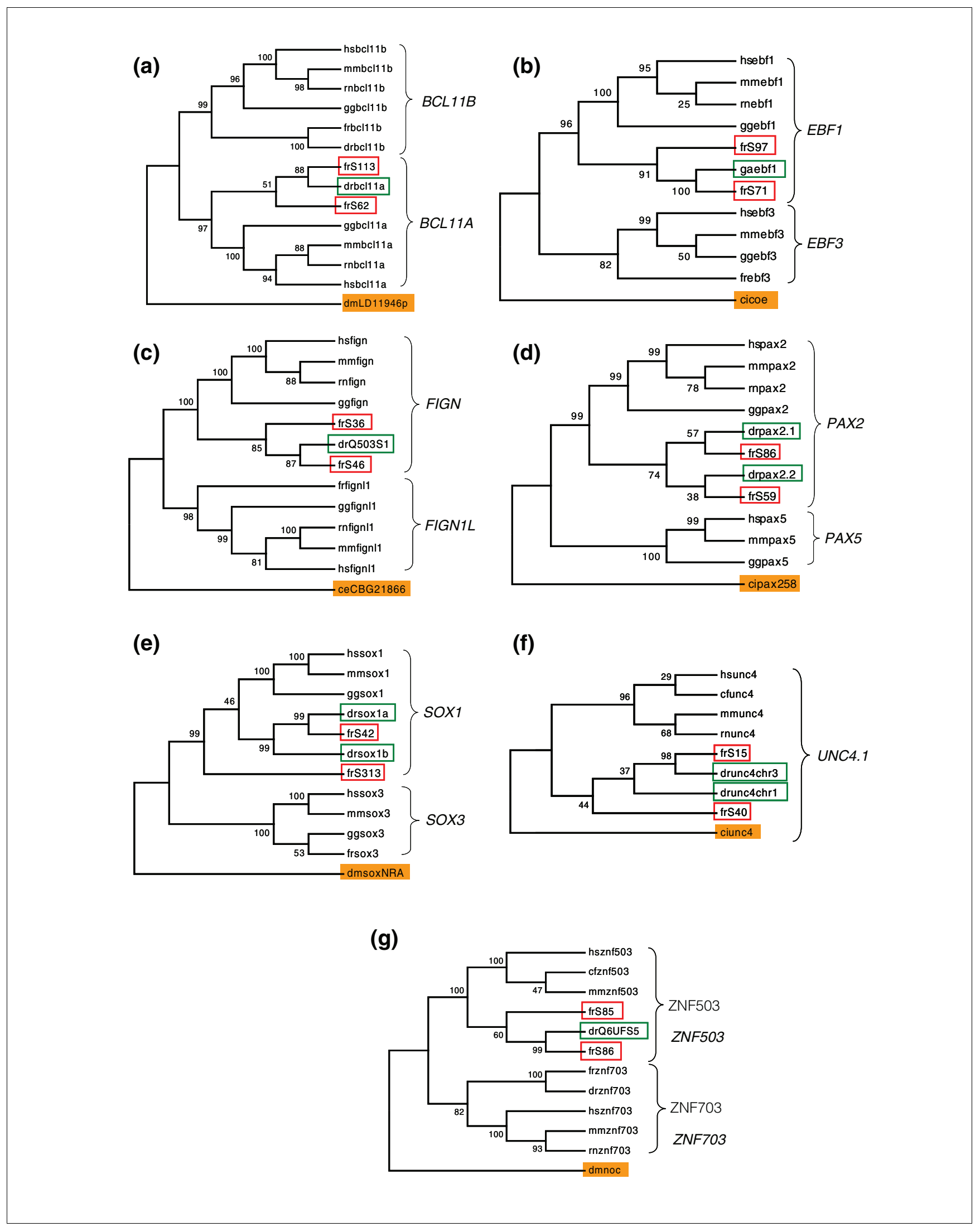

Figure I (see legend on next page) 
Figure I (see previous page)

Phylogenies of seven Fugu co-orthologs. Fugu (fr) co-ortholog protein sequences are highlighted by red boxes and named according to scaffold number they were located on (for example, frS86 = scaffold_86). Zebrafish (dr) or stickleback (ga) sequences are highlighted by green boxes and uncharacterized proteins named after the SwissProt ID or the chromosome they are located on. Bootstrap values are indicated at each node. Other tetrapod sequences included: human (hs), mouse ( $\mathrm{mm})$, rat $(\mathrm{rn}), \mathrm{dog}(\mathrm{cf})$ and chicken $(\mathrm{gg})$. Invertebrate outgroups are shaded orange and contain sequences from the following species: Ciona intestinalis (ci), Drosophila melanogaster (dm) and Caenhoribditis elegans (ce). Trees: (a) BCLI IA using the closest paralog BCLI IB as a comparator. (b) EBFI using the closest paralog EBF3 as a comparator. (c) FIGN using the closest paralog FIGNIL as a comparator. (d) PAX2 using one of its two closest paralogs PAX5 as a comparator. (e) SOXI using its closest paralog SOX3 as a comparator. (f) UNC4.I has no known closely related paralogs. (g) ZNF503 using its closest paralog ZNF703 as a comparator.

\section{CNE distribution and changes in genomic environment around Fugu co-orthologs}

CNEs were independently identified within each Fugu coorthologous region by carrying out a combination of multiple and pairwise alignment with the same orthologous sequence from human, mouse and rat (the entire dataset from this study can be accessed and queried through the web-based CONDOR database [53]). The regions in which CNEs were located for each co-ortholog together with surrounding gene environment can be seen in Figure 2.

All but one of the CNE regions in human are located in genepoor regions termed 'gene deserts' that flank or surround the trans-dev gene and are characteristic of regions thought to contain large numbers of cis-regulatory elements [30]. These gene deserts appear to have been conserved to some degree in both Fugu copies (albeit in a highly compact form). For example, a large gene desert of approximately 2.2 $\mathrm{Mb}$ is located downstream of $B C L 11 A$ up to the ubiquitin ligase gene FANCL in human, and similar (compacted) versions of this gene desert are present in both Fugu regions, although downstream of bcl11a.2 it is almost a quarter of the size com- pared to the same region in bcl11a.1 (98 kb versus $380 \mathrm{~kb}$ ). In the majority of regions under study (five out of seven), CNEs extend purely within these large intergenic regions directly flanking or within the introns of the trans-dev gene. In those regions in which CNEs extend beyond or within the genes neighboring the trans-dev gene (that is, bcl11a.1, znf5o3.1 and znf503.2) the gene order and orientation between Fugu and human has remained largely conserved, spanning three to five genes, something that is relatively rare within the Fugu genome $[54,55]$. This may be due to functional constraints on these regions whereby it is necessary to maintain the CRM and associated gene in cis [34,56]. For the remaining coorthologous regions the degree of synteny varies widely. For instance, neither Fugu pax2 region has conserved gene order with the human genome. Two orthologs of NDUFB8 and HIF1AN (upstream of human PAX2) are partitioned and rearranged so that hifian is downstream of pax2.1 and ndufb8 is downstream of pax2.2 (Figure 2).

The preservation of $98.5 \%$ of the CNEs (796/811) as well as both trans-dev genes in the same orientation and order along

Table I

Co-ortholog nomenclature and genomic locations in the Fugu genome

\begin{tabular}{|c|c|c|c|c|c|}
\hline Human gene* & Co-ortholog name ${ }^{\dagger}$ & Fugu scaffold $(\mathrm{S})$ location $(\mathrm{kb})^{\ddagger}$ & Length $(\mathrm{kb})^{\S}$ & Prop 'N's (\%) & Fugu protein accession code $\mathrm{e}^{¥}$ \\
\hline \multirow[t]{2}{*}{$B C L I / A$} & bcll la.l & SII3: $|40.8-5| 8.9$ & 378.1 & 2.98 & NEWSINFRUP00000I42044 \\
\hline & $b c l l \mid a .2$ & S62: $603.7-740.4$ & 136.7 & 0.18 & NEWSINFRUP00000I44873 \\
\hline \multirow[t]{2}{*}{ EBFI } & ebfl.I & S97: $400.4-483.3$ & 82.9 & 0.82 & NEWSINFRUP00000I 27762 \\
\hline & ebfl.2 & S7I: $999.3-1,091.7$ & 92.4 & 1.90 & NEWSINFRUP00000I48373 \\
\hline \multirow[t]{2}{*}{ FIGN } & fign.l & S36: $382.6-486.8$ & 104.2 & 0.16 & NEWSINFRUP00000I53680 \\
\hline & fign.2 & S46: $126.9-219.9$ & 93 & 0.39 & NEWSINFRUP00000I7797। \\
\hline \multirow[t]{2}{*}{ PAX2 } & pax2.I & S86: $541.7-669.8$ & 128.1 & 0.29 & - \\
\hline & pax2.2 & S59: 768.9-898.3 & 132.7 & 3.59 & - \\
\hline \multirow[t]{2}{*}{ SOXI } & soxl.l & S42: $1,020-1,105$ & 85 & 1.49 & [Swiss-Prot: Q6WNU3_FUGRU] \\
\hline & sox 1.2 & S3 I 3: 107.2-174.9 & 67.7 & 8.9 & [Swiss-Prot: Q6WNU2_FUGRU] \\
\hline \multirow[t]{2}{*}{ UNC4.I } & unc4.I.I & SI5: 76I.I-825.5 & 61 & 0.32 & NEWSINFRUP00000I54395 \\
\hline & unc4.1.2 & S40: $1,435-1,537$ & 102 & 0.96 & NEWSINFRUG00000161008 \\
\hline \multirow[t]{2}{*}{ ZNF503 } & znf503.I & S86: 7-220 & 213 & 3.64 & NEWSINFRUP00000I8I530 \\
\hline & znf503.2 & S59, S29 (all) & 148.5 & 3.22 & NEWSINFRUP00000I8I454 \\
\hline
\end{tabular}

*Name of human gene ortholog. †Nomenclature of novel Fugu co-orthologs. $\neq$ Location and extent of Fugu genomic scaffold used in multiple alignment. §Length of Fugu genomic region used in multiple alignment. TProportion of Fugu genomic region that is made up of unfinished sequence (that is, runs of 'N's). ${ }^{*}$ The protein accession code for each co-ortholog. These were derived either from Ensembl (v40.4b) or from SwissProt. Protein sequences for pax2.I and pax2.2 were incomplete in both Ensembl and SwissProt and were reconstructed using alignments of full-length amino acid sequences from other species. 


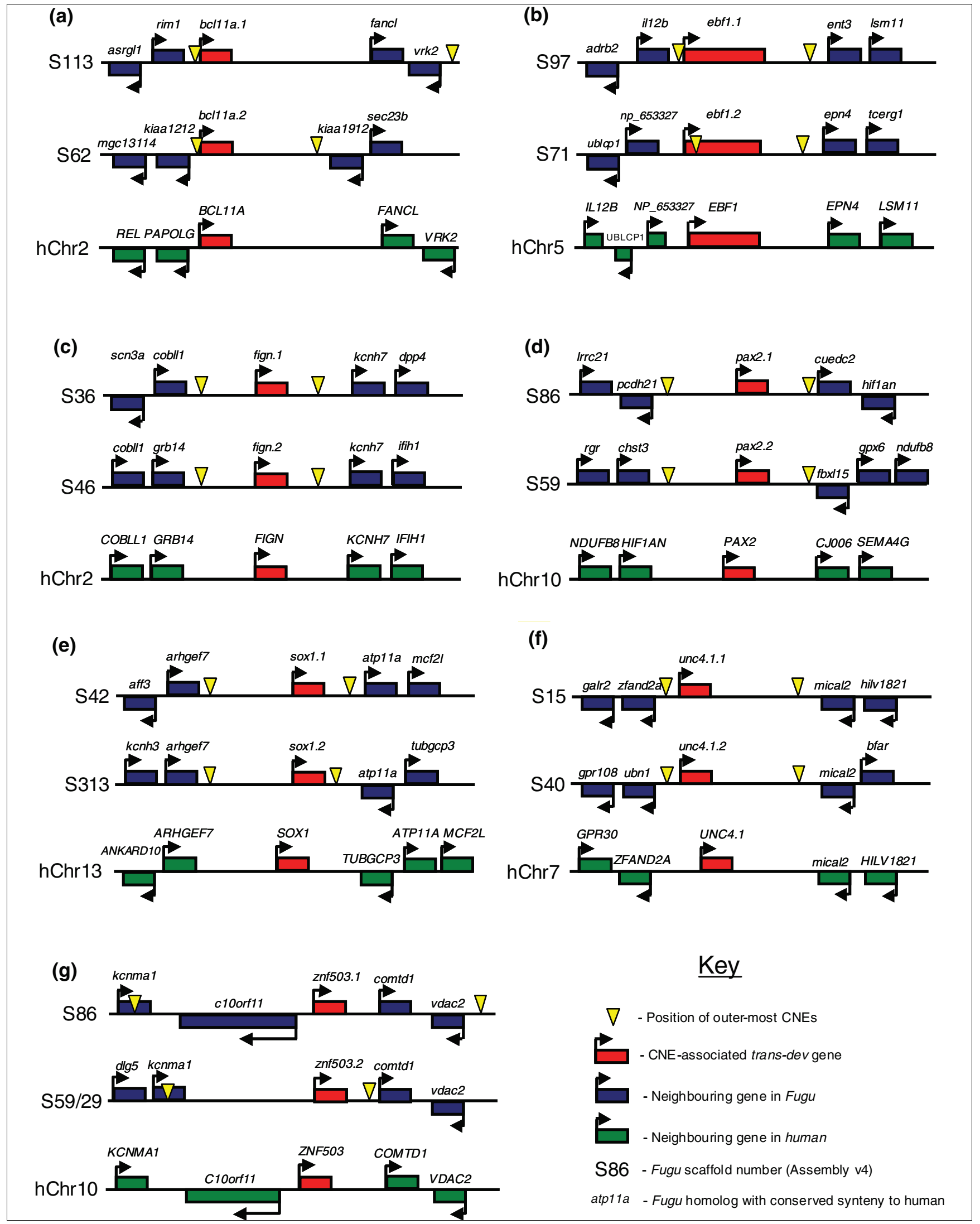

Figure 2 (see legend on next page) 
Figure 2 (see previous page)

Genomic environment around Fugu co-orthologs in comparison to the human ortholog. Diagrammatic representation of the genomic environment around Fugu co-orthologs and human orthologs of: (a) BCLI IA, (b) EBFI, (c) FIGN, (d) PAX2, (e) SOXI, (f) UNC4.I and (g) ZNF503. For each gene, the top two lines represent the genic environment around each of the Fugu co-orthologs whilst the third line represents the genic environment around the human ortholog. Regions are not drawn to scale and are representative only. Human chromosome locations and Fugu scaffold IDs are stated to the left of each graphic. Fugu scaffold IDs can be cross-referenced for their exact location through Table I. All annotation was retrieved from Ensembl Fugu (v36.4) and Human (v.36.35i). Only genes that are conserved in both Fugu and human are shown. Reference trans-dev genes are colored in red and are always orientated in $5^{\prime} \rightarrow 3^{\prime}$ orientation. Surrounding genes in Fugu are marked in blue and in human in green. The names of neighboring Fugu homologs that share conserved synteny with human (but not necessarily the same relative order or orientation) are highlighted in an orange box. Genes orientated in the same direction as the reference trans-dev gene are located above the line and those orientated in the opposite direction are below the line. Yellow triangles represent the positions of the furthest CNEs upstream and downstream in each genomic sequence and delineate the region in which CNEs were identified.

the sequence between human and Fugu, in contrast to the rearrangement of surrounding genes, confirms the likelihood that the CNEs and trans-dev genes identified are associated with each other.

\section{Pattern of CNE retention/partitioning between co- orthologs}

The DDC model for the retention of gene duplicates over evolution states that following duplication, genes undergo complementary degenerative loss of subfunctions or, on the regulatory level, expression domains. Based on the assumption that CNEs represent putative autonomous CRMs that control gene expression to one or more specific expression domains, we would predict that this process of regulatory subfunctionalization would involve the degeneration or loss of these elements between gene duplicates so that the ancestral CRMs were to some degree partitioned between the two genes. We identified 811 CNEs in total for all 14 regions in Fugu with lengths ranging from 30-562 bp (mean = 117 bp, median $=85 \mathrm{bp}$ ) and human-Fugu percent identities ranging from $60-94 \%$ (mean $=74 \%$ ). CNEs from each co-ortholog were defined as 'overlapping' if there was conservation between them to at least part of the same single sequence in human. CNEs that were conserved between human and only one Fugu co-ortholog with no significant overlap to CNEs in the counterpart co-ortholog were defined as 'distinct'. Figure 3 illustrates the definition of overlapping and distinct CNEs identified in a multiple alignment between Fugu regions around pax2.1 and pax2.2, against the reference human $P A X 2$ region.

Similar to other trans-dev gene regions identified previously (for example, [26]), the co-orthologs under study have highly variable numbers of CNEs conserved in their vicinity, ranging from 11 CNEs in sox1.2 to 156 in znf503.1 (Figure 4). Comparison of the overall number of CNEs conserved between coorthologous copies revealed three sets, bcl11a.1/2, ebf1.1/2 and znf503.1/.2, that have notably different overall numbers of CNEs located in their vicinity, indicating a large-scale loss of elements in one co-ortholog compared to its counterpart since duplication (Figure 4). In the cases of bcl11a.1/2 and znf503.1/2, this large-scale asymmetrical loss of elements in one co-ortholog copy correlates to a large decrease in genomic sequence within the same region (Additional data file 2).
Many of the co-orthologs have also undergone substantial partitioning of elements, as indicated by the large proportion of the identified CNEs classified as 'distinct' in each coortholog. For example, fign.1 and fign.2 have a similar number of CNEs in their vicinity (47 and 50, respectively) but $42 \%$ and $56 \%$ of these CNEs, respectively, are distinct to each co-ortholog. The extent of distinct CNEs as a proportion of total CNEs differs significantly between sets of co-orthologs, ranging from $24.5 \%(13 / 53)$ in pax2.1 to $83 \%(34 / 41)$ in $e b f 1.1$ (Figure 4). For co-orthologs of $B C L 11 A$ and $E B F 1$ the majority of CNEs in both genes are distinct. Only in co-orthologs of $P A X 2$ are the majority of CNEs in both genes found to be overlapping (Figures 3 and 4), suggesting a high level of retention of regulatory domains in both genes since duplication. In the majority of gene pairs, namely co-orthologs of $F I G N, S O X 1, U N C 4.1$ and $Z N F 503$, one copy has the majority of its CNEs as distinct while the other has a majority of its CNEs overlapping with that of its counterpart co-ortholog, suggesting an asymmetrical rate of element partition.

The accuracy of these results depends heavily on ensuring that the loss of elements in one co-ortholog is the result of subfunctionalization rather than lack of sequence coverage in the genomic sequence. The proportion of 'N's (sections of unfinished sequence) within each Fugu genomic sequence can be seen in Table 1. We found that only one of the gene regions, sox1.2, contains a significant proportion of unfinished sequence (8.9\%), suggesting some of the CNEs defined as 'distinct' in sox1.1 may have overlapping counterparts in sox1.2. However, closer examination of the positioning of the unfinished sequence reveals that the vast majority occurs in a region easily defined by two flanking overlapping CNEs that contains just a single distinct $\mathrm{CNE}$ in its counterpart coortholog. The region in sox1.2 potentially containing counterparts to most of the distinct CNEs in sox1.1 contains less than $3 \%$ unfinished sequence, suggesting most, if not all, of these distinct CNEs are defined correctly. Without 100\% finished sequence in all cases it is, of course, possible that a small proportion of the CNEs identified as distinct in these coorthologs may have an overlapping counterpart within unfinished sequence, but given the high levels of finished sequence in most of the gene regions, this is unlikely to account for a significant number. 


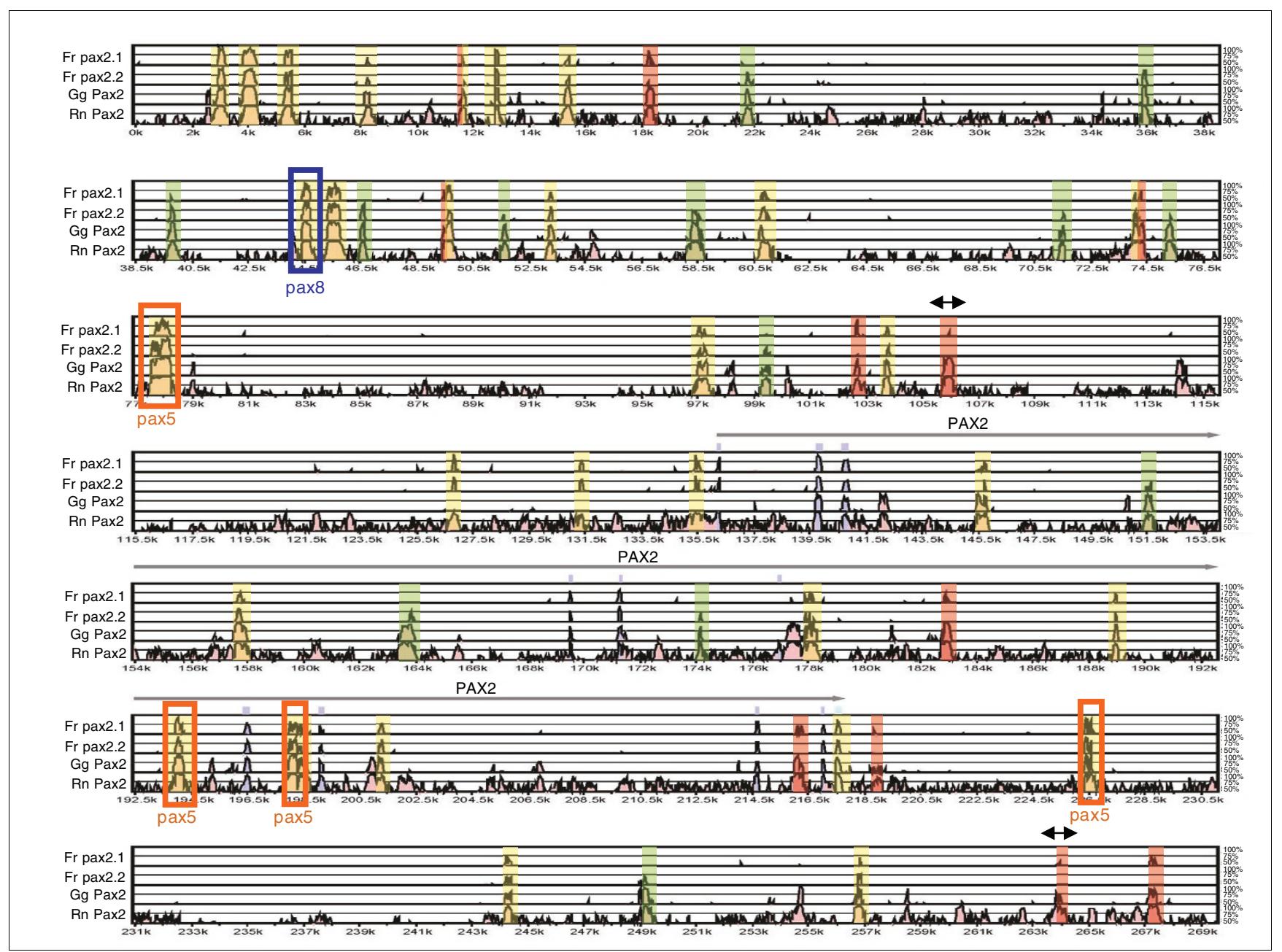

Figure 3

VISTA plot of an MLAGAN alignment of orthologous regions surrounding two pax2 co-orthologs in Fugu ( $\mathrm{Fr}$ ) and Pax2 in chicken (Gg), rat (Rn) and human. The baseline is $268 \mathrm{~kb}$ of human sequence. Conservation between human and each sequence is shown as a peak. Peaks that represent conservation in a non-coding region of at least $65 \%$ over 40 bp are shaded pink with coding exons shaded purple and peaks located within untranslated regions shaded light-blue. All CNEs conserved in at least one of the Fugu co-orthologs are color-coded. CNEs in both Fugu co-orthologs that overlap the same region in human are shaded yellow while CNEs that are 'distinct' (or conserved solely) in pax2.I are shaded red and CNEs distinct to pax2.2 are shaded green. Peaks marked with a double-headed arrow are conserved in Fugu in the opposite orientation (and therefore do not show up in the VISTA plot). A number of the CNEs around PAX2 are also duplicated CNEs (dCNEs) that are located elsewhere in the genome in the vicinity of PAX2 paralogs. CNEs marked with an orange box have another $\mathrm{dCNE}$ family member in the vicinity of PAX5 and the CNE marked with a blue box has a dCNE family member conserved upstream of PAX8.

\section{Evolution of overlapping CNEs since duplication}

Overlapping CNEs comprise a large proportion and, in some cases, the majority of CNEs identified around many of the gene pairs and have, therefore, remained to some extent under positive selection in both co-orthologs. The distribution of lengths and percent identities for 381 overlapping CNEs versus 430 distinct CNEs is significantly different for both lengths $\left(p<1 \times 10^{-16}\right)$ and percent identities $\left(p=1.1^{-8}\right)$. Overlapping CNEs have significantly higher average lengths (mean $=149.6 \mathrm{bp}$, median $=116.1 \mathrm{bp})$ than distinct CNEs (mean $=87.6 \mathrm{bp}$, median $=62 \mathrm{bp}$ ) as well as slightly higher percent-identities (mean $=75.2 \%$ and median $=75 \%$ for overlapping versus mean $=72.4 \%$ and median $=71.7 \%$ for distinct). Only 4 of the distinct CNEs overlap to some degree but by less than the arbitrary $20 \mathrm{bp}$ cut-off required for CNEs to be defined as overlapping. Removing these leaves the mean lengths and percent-identities virtually unchanged, confirming that the cut-off did not significantly bias the distribution of distinct elements towards smaller elements.

We studied two aspects to gauge evolutionary changes occurring in these elements since duplication: changes in element length and changes in substitution rate between overlapping CNEs in Fugu.

\section{CNE length}

A total of 182 pairs of overlapping CNEs were identified across all co-ortholog pairs with a one-to-one relationship. 


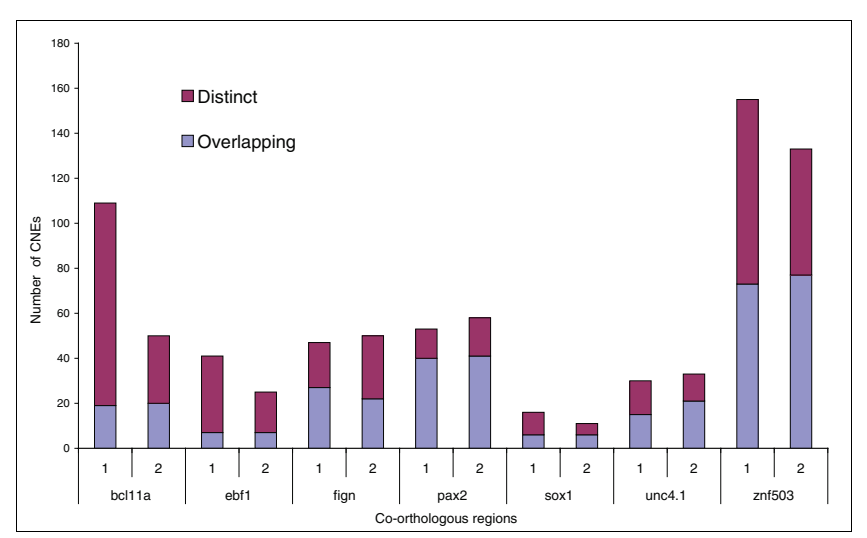

Figure 4

Proportion of CNEs around each Fugu co-ortholog that overlap or are distinct to sequences in mammals compared to CNEs identified in its counterpart co-ortholog. Each bar represents the total number of CNEs identified around each co-ortholog with a proportion of that total colored as overlapping (light purple) or distinct (maroon) CNEs.

The length of the overlap in the human sequence between coorthologous CNEs ranged from 24-46o bp (mean = 107.5 bp \pm 2.27 standard error of the mean). For each overlapping pair, we calculated the proportion of the overlapping sequence as a function of the full length Fugu-human conserved sequence in each co-ortholog. We found $62 \%$ of the pairs to have undergone significant degeneration in element length in one of the copies compared to its counterpart (Figures 5 and 6); 30\% of pairs overlapped over the majority of both elements, suggesting little evolution of element length since duplication, and approximately $8 \%$ have undergone a significant level of degeneration in element length in both copies at their edges. These results suggest the process of subfunctionalization may also be occurring, at least in some of these cases, through the partial loss of function in both copies, allowing gene preservation through quantitative complementation (as suggested in [7]). It is also possible that sequence loss could causes changes in module function through the change in binding site combinations present. In genes such as pax2.1 and pax2.2 that have the majority of their CNEs overlapping in both genes, this presents an additional mechanism by which both copies may be preserved. In addition to overlapping CNEs that have undergone evolution at their edges, 29 overlapping CNEs have undergone evolution at the centre of the element, essentially creating a split element (that is, a CNE in one co-ortholog overlaps two or more CNEs from the other co-ortholog).

\section{CNE sequence evolution}

Overlapping CNEs are conserved to the same human sequence across the length of the overlap. However, it is possible that elements have undergone differential evolution, with one element containing a significantly greater number of independent substitutions than the other, indicative of either subfunctionalization or neofunctionalization. To measure whether the sequence of one CNE has diverged faster than its counterpart, we used the Tajima relative rate test [57] with the human sequence as the outgroup (or ancestral) sequence. The Tajima relative rate test measures the significance in the difference of independent substitutions in each sequence relative to the outgroup sequence using a chi-squared statistic (see Additional file 3 for the results of relative rate tests for all overlapping CNEs). The percentages of overlapping CNEs that show a statistically significant difference in substitution rate in one copy over another range from $17 \%$ in sox 1 to $26 \%$ in znf5O3 (Table 2). One of the most significant examples within this set was found in a pair of CNEs upstream of coorthologs of UNC4.1 and can be seen in Figure 6. These results suggest that a substantial number of the elements appear to have undergone an asymmetrical rate of evolution since duplication, something we would expect under the DDC model. Alternatively, if these changes were positively selected it may indicate a process of neofunctionalization whereby coorthologs have evolved novel regulatory patterns to that of the ancestral copy.

\section{A history of duplications: some co-orthologous CNEs were duplicated in ancient events at the origin of vertebrates}

In addition to being involved in a teleost-specific duplication event, a number of the CNEs identified around the trans-dev genes in this study have been previously retained from ancient duplications thought to have occurred at the origin of vertebrates. While the majority of CNEs are single copy in the human genome, a recent study identified 124 families of CNEs genome-wide that have more than one copy across all available vertebrate genomes and are referred to as 'duplicated CNEs' (dCNEs) [29]. dCNEs are associated with nearby trans-dev paralogs and a number have been shown to act as enhancers that drive in vivo reporter-gene expression to similar domains [29]. The absence of these sequences in nonvertebrate chordate genomes and their association with paralogs that arose from whole-genome duplication events at the origin of vertebrates [58] places their origins sometime prior to this event more than 550 million years ago. The conservation of these elements over such extreme evolutionary distances suggests they play critical roles in the regulation of paralogs that have since undergone neofunctionalization. We found 30 non-redundant human CNEs (conserved to 52 coorthologous CNEs in Fugu) to be dCNEs in the vicinity of one or more paralogs of the nearby trans-dev gene (Table 3). This further confirms the tight association of these CNEs with their nearby trans-dev genes as dCNEs resolve the CNE-gene association more clearly [59]. These dCNEs were identified in five of the seven co-orthologous regions with some dCNEs associated with more than one paralog (for example, $P A X 2$ associated dCNEs located in the vicinity of $P A X 5$ and $P A X 8$; Table 3; Figure 3). 80\% of the co-ortholog CNEs identified as dCNEs $(42 / 52)$ are conserved in both co-ortholog regions in Fugu, a two-fold enrichment ( $p<0.001$ ) over the expected number given the overall proportions of overlapping and distinct elements in the CNE dataset. 


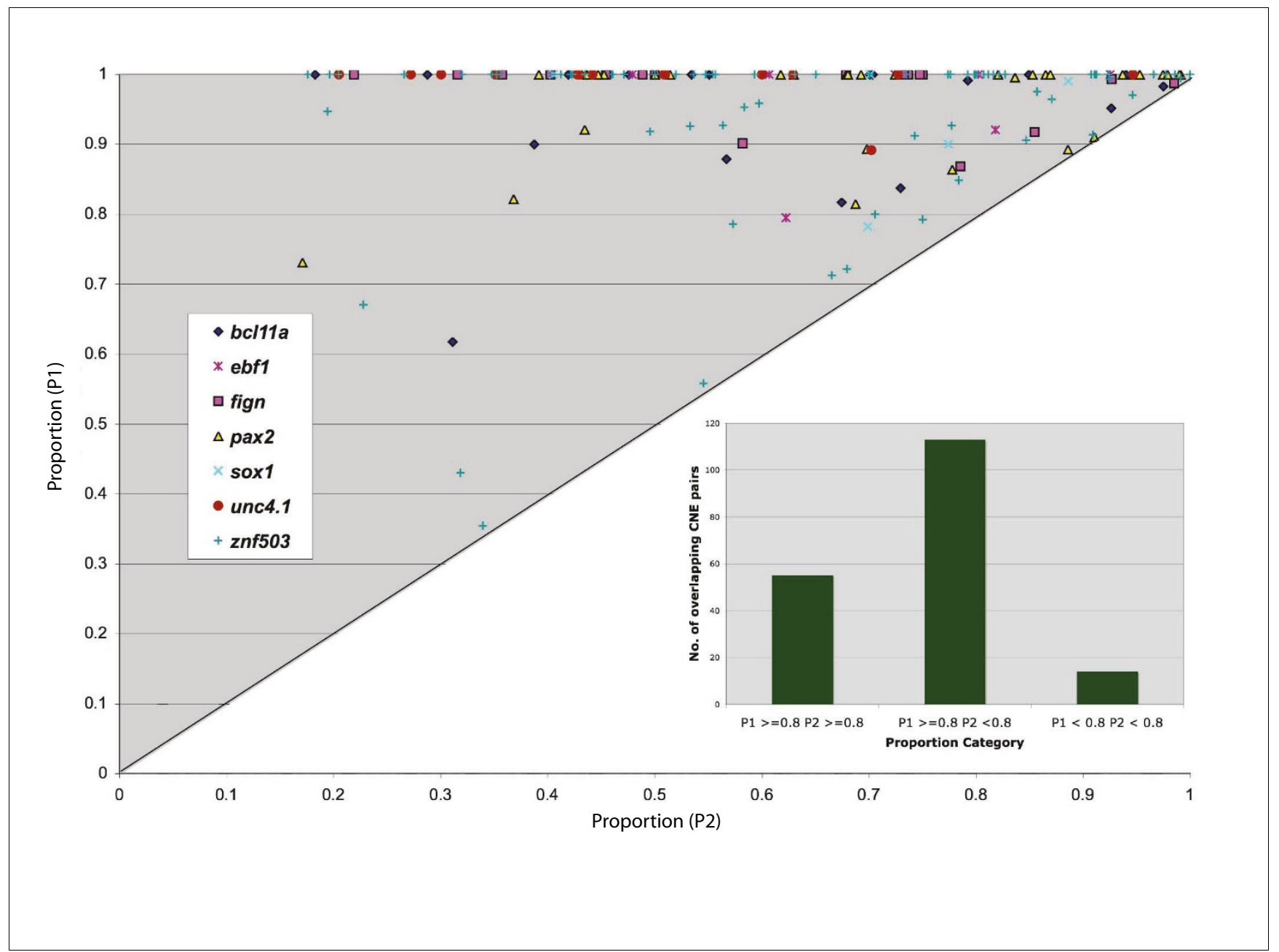

Figure 5

Proportion of each CNE sequence that overlaps the counterpart co-ortholog CNE. Main graph: for each overlapping pair of co-orthologous CNEs (involving just two sequences), the proportion of the full length of each CNE (PI-P2) made up by the overlap was calculated using the human sequence as the reference. The larger of the two proportions was always plotted as PI to simplify analysis. Inset bar chart: summary of the number of overlapping CNE pairs falling into three main proportion categories: $\mathrm{PI} \geq 0.8, \mathrm{P} 2 \geq 0.8$ - pairs that overlapped over the majority of both elements, suggesting little evolution of element length since duplication; $\mathrm{PI} \geq 0.8, \mathrm{P} 2<0.8$ - pairs that have undergone significant degeneration in element length in one of the copies compared to its counterpart; $\mathrm{PI}<0.8, \mathrm{P} 2<0.8$ - pairs that have undergone a level of degeneration in element length in both copies at their edges.

\section{Discussion}

Recent studies show there are a surprisingly large number of duplicated genes present in the genomes of all organisms that cannot be accounted for by the classic models of nonfunctionalization and neofunctionalization. The presence of large numbers of duplicated genes within the genomes of teleost fish, now widely presumed to have undergone a whole genome duplication event around 300-350 million years ago, provide an excellent opportunity for comparative studies to test the DDC model. Prior to the availability of large-scale genomic sequences, the ability to study regulatory subfunctionalization through identifying the regulatory elements responsible was limited due to a lack of appropriate identification strategies. The discovery of thousands of CNEs con- served across the vertebrate lineage, highly enriched for sequences likely to be distal cis-regulatory modules, allowed us to develop a strategy to begin to uncover this. We identified potential gene candidates that contain both CNEs in their vicinity and are likely to derive from fish-specific duplication events using data from the initial whole genome comparison of the Fugu and human genomes. CNEs that cluster in the same location in human but derive from two separate locations in the Fugu genome strongly indicate the presence of coorthologous regions. We selected seven clusters of CNEs in the human genome, each in the vicinity of a single trans-dev gene that fulfilled these criteria. For each of these genes, we recreated a phylogeny using protein sequences identified in each Fugu region, confirming the genes are both orthologs 


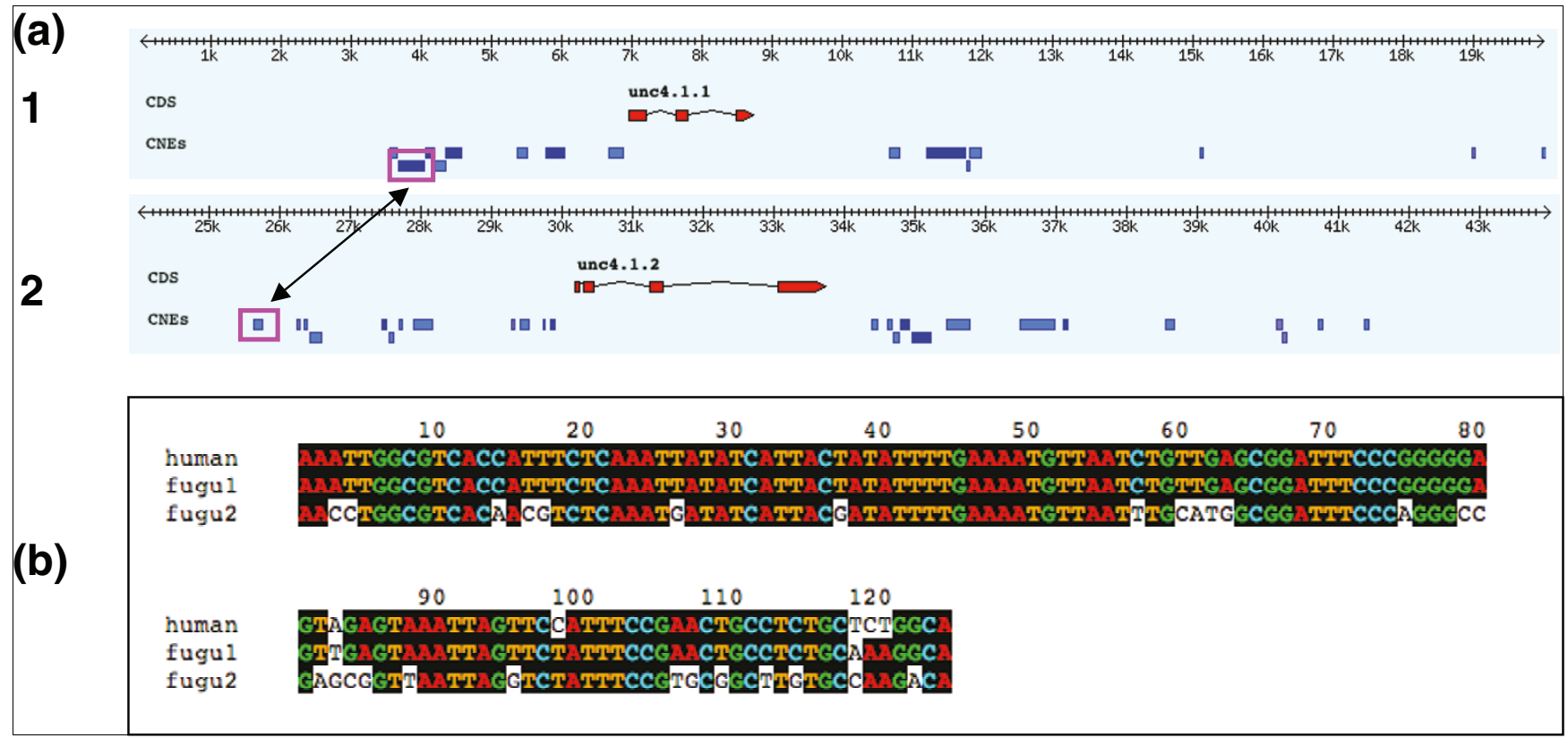

\section{Figure 6}

Significant change in element length and substitution rate in overlapping CNEs upstream of unc4.I.I and unc4.I.2. (a) CNEs (filled blue boxes) were identified around each Fugu co-ortholog unc4.I.I (AI, top) and unc4.I.2 (A2, bottom) (gene exons are shown in the coding sequence (CDS) track as filled red boxes). The scale at the top represents positions along the Fugu sequence used in the multiple alignment. Two CNEs, highlighted in pink boxes, one upstream of Fugu unc4.I.I (CRCNEAC0003 I954 [53], referred to as CNE_AI) and one upstream of unc4.I.2 (CRCNEAC00032205 [53], referred to as CNE A2) are conserved to part of the same sequence in human upstream of UNC4.I. The overlap region is 126 bp in length and encompasses all of the CNE_A2 but only $35 \%$ of CNE_AI (which is 360 bp long), indicating a significant loss of element length in CNE_A2. (b) A relative rate test of the Fugu CNEs across the overlapping region using human as the outgroup reveals a highly significant number of independent substitutions (26) in CNE_A2 with no independent substitutions in CNE_AI $(p<0.00 I)$. This suggests CNE_Al is likely to have retained the ancestral function while CNE_A2 may have evolved to have a different function.

(co-orthologs) of the single mammalian copy, and all topologies confirmed the genes derive from a duplication event following the split between ray-finned and lobe-finned fishes. This relationship was further confirmed by comparison of the genic environment around the trans-dev gene in both Fugu regions to that of the single region in human. Conserved gene

Table 2 order extends, in many cases, to one or more genes upstream and/or downstream of each co-ortholog, indicating a shared ancestral origin, although in several instances the neighboring genes have been partitioned between the co-orthologous regions, undergone rearrangement or have been lost.

\section{Tajima relative rate tests of overlapping co-orthologous CNE in Fugu}

\begin{tabular}{|c|c|c|c|c|c|}
\hline \multirow[t]{2}{*}{ Gene region* } & \multirow[t]{2}{*}{ No. of overlapping pairs ${ }^{\dagger}$} & \multirow[t]{2}{*}{ No. of CNE pairs with $p>0.05 \ddagger$} & \multicolumn{2}{|c|}{ No. of CNE pairs with $p \leq 0.05 \S$} & \multirow[t]{2}{*}{$\%$ of CNE pairs with $p<0.05 \%$} \\
\hline & & & Co-ortholog I & Co-ortholog 2 & \\
\hline$B C L I / A$ & 20 & 15 & 1 & 4 & 25 \\
\hline$E B F I$ & 7 & 5 & I & I & 21 \\
\hline FIGN & 28 & 21 & 5 & 2 & 25 \\
\hline PAX2 & 43 & 34 & 4 & 5 & 21 \\
\hline SOXI & 6 & 5 & 0 & 1 & 17 \\
\hline UNC4.I & 20 & 15 & 0 & 5 & 25 \\
\hline ZNF503 & 84 & 62 & 5 & 17 & 26 \\
\hline
\end{tabular}

*Gene region. TTotal number of overlapping CNEs within gene region. $¥$ Numbers of overlapping CNE pairs with no significant difference in substitution rates (that is, $p$ values of $>0.05$ ). $\$$ The number of overlapping CNEs that exhibit a significant difference in substitution rate (that is, $p$ value $\leq 0.05$ ) in the CNE sequence in the vicinity of one co-ortholog over that in the other. IThe percentage of overlapping CNEs with significantly different substitution rates in either co-ortholog as a proportion of the total number of overlapping CNEs. 
Table 3

\begin{tabular}{|c|c|c|c|}
\hline Gene region & Co-ortholog I & Co-ortholog 2 & $\begin{array}{l}\text { Gene paralog in the vicinity of the } \\
\text { dCNE(s) }\end{array}$ \\
\hline \multirow[t]{6}{*}{$B C L I I A$} & CRCNE00002445 & CRCNE000046I 4 & $B C L L I B$ \\
\hline & CRCNE00002557 & - & \\
\hline & CRCNE00002548 & CRCNE00004648 & \\
\hline & CRCNE00002544 & CRCNE0004643 & \\
\hline & & CRCNE0004644 & \\
\hline & CRCNE00002540 & - & \\
\hline \multirow[t]{5}{*}{ EBFI } & CRCNE000I077I & - & EFB3 \\
\hline & - & CRCNE000I08I8 & \\
\hline & CRCNE00000027 & CRCNE000I0823 & \\
\hline & CRCNE000I0778 & CRCNE000I0827 & \\
\hline & CRCNE000I0787 & - & \\
\hline EBFI & CRCNE000I0772 & CRCNE000I0820 & $E B F I / 2 / 3 / 4$ \\
\hline PAX2 & CRCNE00000064 & CRCNE00000I33 & PAX8 \\
\hline \multirow[t]{4}{*}{ PAX2 } & CRCNE0000007I & CRCNE00000I 47 & PAX5 \\
\hline & CRCNE00000090 & CRCNE00000I65 & \\
\hline & CRCNE00000092 & CRCNE00000I67 & \\
\hline & CRCNE00000099 & CRCNE00000I74 & \\
\hline SOXI & - & CRCNE00001926 & sox2 \\
\hline \multirow[t]{14}{*}{ ZNF503 } & CRCNE000IOII 2 & CRCNE00004977 & ZNF703 \\
\hline & CRCNE000I0I47 & CRCNE00004994 & \\
\hline & CRCNE000I0I 26 & CRCNE00005024 & \\
\hline & CRCNE000I0I67 & - & \\
\hline & CRCNE000IOI 70 & - & \\
\hline & CRCNE000I0I87 & - & \\
\hline & CRCNE000IOI 80 & - & \\
\hline & CRCNE000I0I76 & CRCNE000050I3 & \\
\hline & & CRCNE000050I5 & \\
\hline & CRCNE000I0I65 & CRCNE000050II & \\
\hline & CRCNE000I0I6I & CRCNE00005008 & \\
\hline & CRCNE000I0046 & CRCNE00004906 & \\
\hline & CRCNE000IOI56 & CRCNE00005003 & \\
\hline & CRCNE00010120 & CRCNE00004986 & \\
\hline
\end{tabular}

CNEs in each co-ortholog are referred to by their CONDOR database identifiers [53]. Each CNE was considered duplicated if the human sequence they are conserved to shows significant hit to a sequence elsewhere in the genome through BLAST. Any gene in the vicinity $(<1.5 \mathrm{Mb}$ away) of the BLAST hit that is paralogous to genes within a window of the same size around the query CNE is shown in the final column.

The process of subfunctionalization, as described in the DDC model, is defined as the fixation of complementary loss of subfunctions that result in the joint preservation of duplicate loci [7]. For regulatory subfunctionalization, a subfunction may represent expression of a gene in a specific tissue, cell lineage or temporal stage. For genes with complex regulation these subfunctions are controlled by one or a combination of cis-regulatory modules. A proportion of these can be predicted through the comparative genomic approaches outlined in this study and for the purposes of this discussion are assumed to be represented by CNEs. Under the DDC model, subfunctionalization is thought to occur by two different routes: qualitative and quantitative [7]. Under qualitative subfunctionalization one duplicate copy undergoes one or more complete loss-of-subfunction mutations with the second copy subsequently acquiring null-mutations for a different set of subfunctions. Thus, each copy is required to recapitulate the full set of ancestral subfunctions. Under this route, a conserved element representing an independent cisregulatory module that undergoes a null-mutation in one of the gene copies will no longer be under selective constraint, and will be 'lost' (that is, will not be detectable by sequence conservation) through the accumulation of degenerative mutations over evolution. This process should, therefore, be evident by the effective partitioning of conserved elements between co-orthologs. In contrast, quantitative subfunction- 
alization is more subtle and results from the fixation of reduction-of-expression mutations in both duplicates [7]. Here, both regulatory modules must be maintained in the genome once the summed activity for a particular subfunction in both copies has been reduced to the original level in the single ancestral gene.

By comparing each Fugu co-ortholog with its single orthologous region in mammals, we attempted to identify those 'ancestral' cis-regulatory modules present in the mammalian copy that are retained in only one of the Fugu copies and those that have to some extent been retained in both copies. This approach is particularly appropriate for early developmental regulators for which function and regulation are likely to be highly constrained across all vertebrates and the mammalian gene represents as close as possible the ancestral preduplication state. The probability of the preservation of gene duplicates through subfunctionalization is also assumed to be higher in genes with complex regulation that contain large numbers of independently mutable CRMs [6o], a view reinforced by the overrepresentation of genes involved in development and cellular differentiation found in fish-specific gene duplicates [38]. As with trans-dev genes across the genome, overall numbers of CNEs between gene pairs differs substantially and is likely to reflect differences in regulatory complexity or the extent to which regulation in these genes has been conserved across vertebrate evolution. All seven pairs of co-orthologs contained a number of CNEs that have been partitioned into each co-ortholog along the lines of the qualitative subfunctionalization model. The extent to which CNEs have been partitioned between co-orthologs, however, varies widely. For some co-ortholog pairs, such as bcl11a and $e b f 1$, the majority of CNEs appear to be completely partitioned between the two genes, whilst for other pairs, such as pax2, only a relatively small proportion is. In the DDC model, following initial subfunctionalization, the process of nullmutation fixation in persisting redundant subfunctions is thought to be random, leaving a roughly equal number of subfunctions in each gene copy. Although this appears to be true for the fign, pax2 and unc4.1 co-orthologs, partitioning in bcl11a.1/.2 and $e b f 1.1 / .2$ is highly asymmetrical. In both of these cases there is relatively little overlap between the complement of CNEs associated with each co-ortholog pair. It is possible, therefore, that the loss of some CNEs in one coortholog may have consequences for further loss of elements in that gene. CRMs may not all be functionally autonomous and may interact together to actuate their regulatory role [61]. The degeneration of one or more integral CRMs from a coortholog could accelerate further degeneration of other CRMs that are functionally dependant on them. Under this scenario, a gene duplicate may undergo substantial loss of elements, possibly influencing further asymmetrical loss.

In addition to CNEs that have undergone full partitioning between co-orthologs, some pairs have also retained a number of overlapping CNEs that have been preserved to some extent in both copies. For co-orthologs such as pax2.1/ pax2.2, this type of CNE constitutes the majority of CNEs located around these genes, and is a common feature in most of the other co-ortholog pairs. Overlapping CNEs appear, in general, to be longer than distinct CNEs, although at this stage the relationship (if any) between element size/conservation and its functional importance/regulatory complexity is still unknown. While some of these elements have remained virtually identical in length since duplication, others have undergone major changes both at the edges and at the core of one of the elements, suggesting information loss in one or both copies. A significant proportion of overlapping CNEs have also undergone asymmetrical rates of substitution, suggesting one copy retained the ancestral function while the other was free to evolve, possibly to a novel function.

What explanations could account for this high level of CNE retention observed between co-orthologs? The first is the possibility that some CNEs have undergone quantitative subfunctionalization. Here, degenerative mutations in both CRMs lead to a partial loss of subfunction in each element (such as a reduction in the level of expression in a specific tissue) rather than complete loss. Therefore, both elements must be maintained in the genome once the summed activity for a particular subfunction in both copies has been reduced to the original level of the ancestral gene [7,6o]. Alternatively, some of these elements may function as silencers or insulators or play roles in chromatin remodeling, ensuring correct regulatory compartmentalization and control of both gene duplicates. Another explanation could be the possible interrelations of cis-regulatory modules. As previously mentioned, there is a possibility that some CRMs are interrelated and act in concert to perform their function. It is possible, therefore, that the loss of a CRM critical for the function of other CRMs could lead to large-scale loss in one gene copy. For example, the partitioning of two CRMs that are functionally independent but both dependent on another CRM for correct function would lead to the retention of that critical CRM in both gene copies. Finally, it is possible that although both elements have retained general sequence identity, small nucleotide changes between the elements (such as those seen in asymmetrically evolving CNEs) may have substantial consequences for element function. Indeed in a recent pioneering study, Tümpel et al. [62] pinpointed subtle sequence changes within well-defined enhancers responsible for divergent expression of hoxa2 co-orthologs (hoxa2 (a) and hoxa2(b)) in Fugu. Sequences of the enhancers responsible for full expression of HOXA2 in mice and chicken within the hindbrain were found to be generally conserved in both Fugu copies. Nevertheless, it was demonstrated that a small number of base-pair differences between hoxa2(a) and hoxa2(b) enhancers within several known transcription factor binding sites was sufficient to erase enhancer activity and was shown to be responsible for the lack of expression of hoxa2(a) within certain expression domains. However, the authors could not explain why the non-functional enhancers in hoxa2(a) had 
remained partially conserved, although they postulated they may have regulatory roles in expression domains not covered by the survey. This study highlights the power of correlating known expression differences between co-orthologs with comparative sequence analysis, especially with previous knowledge of the binding sites involved. It also highlights, as functional assays on more ancient duplicated CNEs have demonstrated [29], that sequence similarity may not always extend to functional similarity. Indeed, it is equally plausible that some of the sequence evolution seen between some overlapping CNEs is indicative of neofunctionalization. It would be of great interest for future studies to correlate any novel expression of teleost co-orthologs compared to other vertebrate homologs with changes in these elements. Finally, CNEs may represent several independent or overlapping CRMs and the loss of sequences within the CNE may be due to loss of just one of the CRMs, constituting a form of qualitative subfunctionalization

The pattern of CNE retention and evolution in these Fugu coorthologs is certainly consistent with both mechanisms of subfunctionalization inherent in the DDC model. However, the extent of the contribution of each mechanism to subfunctionalization is different for each gene pair. This could be a consequence of each co-ortholog pair having followed a different evolutionary path after duplication; each under a number of different selective pressures depending on their expression and/or function as well as the influence of stochastic evolutionary events following a relaxation of evolutionary constraint due to genetic redundancy. It is clear, therefore, that confirmation of regulatory subfunctionalization in these gene pairs will require both the characterization of expression patterns for both co-orthologs (through approaches such as in situ hybridization) and confirmation of the regulatory potential of their surrounding CNEs through rapid in vivo reporter-gene assays. Currently, due to the limitations of Fugu as an experimental model, none of the expression profiles for the genes in this study have been characterized, which could be used to assess the extent and type of regulatory change these gene duplicates have undergone. In the more commonly used zebrafish experimental model organism gene expression profiles of two gene-pairs from this study, pax2 and sox1, have been characterized. Expression patterns of PAX2 co-orthologs of PAX2 (Pax2a and Pax2b) [63] are highly similar, although absence of Pax2 $b$ expression in the developing kidney as well as differences in temporal expression confirms they have undergone a level of regulatory differentiation. This appears to corroborate the pattern of element retention/partitioning seen in Fugu pax2 co-orthologs, where the majority of CNEs are largely conserved in both copies with a smaller number of elements partitioned between each gene. This suggests that, similar to their zebrafish homologs, they may have largely overlapping expression domains and have undergone a more subtle form of quantitative subfunctionalization through changes in their temporal expression. A recent survey of expression of the SOX B family of genes identified a level of regulatory differentiation between the zebrafish soxia and soxib co-orthologs [64]. The main differences in expression are temporal (for example, soxia expressed in the lens a number of hours before soxib), although there are also spatial differences with soxia expression initiated in hindbrain and forebrain whereas soxıb initiates only in the forebrain. The overall expression patterns of these co-orthologs correspond closely to $S O X 1$ expression in other vertebrates, indicating that changes in their expression are due to subfunctionalization rather than neofunctionalization. Our study reveals that at least half of all CNEs identified around soxi co-orthologs have been partitioned, indicative of a level of subfunctionalization, while only one of the overlapping CNEs has undergone a significant level of substitution; a possible reflection on the lack of neofunctionalization in these genes. As patterns of subfunctionalization are known to occur differently between fish species, it remains for further studies of the pax 2 and sox1 co-orthologs in Fugu to discover whether expression differences are similar to those observed in zebrafish.

The majority of regions in this study, in addition to containing CNEs derived from a teleost-specific duplication have counterparts elsewhere in the genome that derive from more ancient vertebrate-specific duplications, reflecting the complex duplication histories of their associated genes. The fact that most of these sequences are retained not only between co-orthologs (for example, bcl11a.1 and bcl11a.2) but also between out-paralogs (for example, BCL11A and BCL11B) spanning over half a billion years of evolution is an indicator of the potentially critical nature of these sequences to the regulation of these genes. In addition, this dataset provides many candidates for further functional studies on the evolution of these sequences and the implications of these changes on their neofunctionalized paralogs and subfunctionalized co-ortholog targets.

The role the teleost-specific genome duplication has played in the evolution of this lineage remains unclear. It is now generally accepted that the genome duplication event(s) that occurred at the origin of vertebrates played a major role in species diversity and, in particular, the huge increase in vertebrate morphological complexity $[65,66]$. In contrast, the more recent teleost specific genome duplication does not appear to have had the same effect, with arguably less complexity in the teleost anterior-posterior axis than in tetrapods [5]. Speciation in teleosts though is unmatched among descendants of other vertebrate lineages, with over 22,000 known species, making up over half of all extant vertebrates species [67]. This has led to suggestions that the genome duplication event may be directly responsible $[14,68]$. Indeed, evidence presented in a review by Taylor et al. [13] indicates that ployploidized members of the Salmon family and Catostomidae (sucker fish) exhibit higher degrees of speciation than members of the same family that remain diploid. Subfunctionalization has been proposed as a likely mecha- 
nism for this increased rate of speciation since differential resolution of subfunctions in multiple gene pairs would lead to reproductive incompatibility due to a reduction in hybrid fitness [69]. Evidence for such lineage-specific subfunction partitioning has been demonstrated for a small number of genes (for example, divergent expression of soxg in stickleback compared to zebrafish [18]), but large-scale studies will be required to resolve the degree of subfunctionalization that took place before and after divergence within the teleost lineage. Furthermore, if lineage speciation is driven by differential subfunctionalization, we might expect the pattern of CRM evolution and partition/retention for the Fugu genes discussed here to be different to those in other fish species. The recent release of a number of divergent draft teleost genomes, including those of zebrafish, medaka and stickleback, should allow further studies in this direction. Furthermore, the approach and analysis used in this study can be extended for use in any situation where genomic regions surrounding duplicated genes can be compared to an orthologous region that has remained single copy. This may be particularly useful for inter-teleost comparisons, where co-ortholog genes have been differentially retained since the whole-genome duplication prior to the teleost radiation.

\section{Conclusion}

Regulatory subfunctionalization is considered to be a major mechanism for the retention of gene duplicates in the genome. This work provides the first large-scale identification and analysis of putative cis-regulatory elements through comparative genomics between duplicated genes using the Fugu genome as a model. Using seven pairs of fish-specific gene duplicates we showed that all pairs have undergone a level of element partition consistent with one of the main mechanisms proposed for regulatory subfunctionalization. In addition, the regulatory elements in this study may have undergone more subtle levels of subfunctionalization through differential loss of element content and asymmetrical rates of substitution. In addition to presenting this work as an analysis in its own right, the methods in this study can be extended to any similar study in which regions derived from an intragenomic duplication can be compared to one or more related genomes in which the orthologous region has remained single-copy.

\section{Materials and methods \\ Identification of CNE-containing co-orthologous regions in the Fugu genome}

An initial set of 2,330 CNEs with little or no evidence of transcription or RNA secondary structure were identified using a whole-genome comparison of the Fugu (assembly v4) and human genomes (assembly v.36) as described in [29]. CNEs in the human genome were grouped into clusters so that each $\mathrm{CNE}$ was no more than $400 \mathrm{~kb}$ in distance from another $\mathrm{CNE}$ in the cluster. Clusters of CNEs in the human genome made up of hits from two non-contiguous Fugu scaffolds (that is, two separate locations in the Fugu genome) were considered further. Previously, we reported that the genes found closest to CNEs are statistically over-represented for Gene Ontology (GO) annotations [70] relating to transcriptional regulation and/or development (trans-dev) [26]. Genes within each of these clusters in the human genome (including the closest gene either side of the cluster) were considered to be trans$d e v$ if they contained any of these over-represented GO annotations. To avoid ambiguities in associating CNEs to genes, we selected only those regions containing a single trans-dev gene within the CNE cluster. Ten pairs of Fugu scaffolds conformed to these criteria. Seven regions containing the largest number of CNEs in addition to well defined orthologous sequence within each Fugu region (that is, those that contain genes neighboring the CNE cluster) were selected for further analysis. These scaffolds contain CNEs that are conserved in the vicinity of the following trans-dev genes in the human genome: BCL11A, EBF1, FIGN, PAX2, SOX1, UNC4.1 and ZNF5O3. Fugu protein sequences from the corresponding orthologs were obtained from Ensembl Compara (v36), except in the case of $P A X 2$ where only partial sequences were present. In these cases, tBLASTn searches using known protein sequences from zebrafish pax2 co-orthologs pax2.1 (SPTR: PAX2_BRARE) and pax2.2 (SPTR: O93370) were used to identify the Fugu protein sequence.

To verify that these genes were phylogenetically co-orthologous to mammalian copies we carried out multiple alignments of each pair of Fugu proteins together with available orthologs from human, mouse, rat, dog and chicken using CLUSTALW (v1.83) [71] downloaded from Ensembl Compara (v36) unless otherwise stated. In addition we used all available orthologs from zebrafish. Two of these are previously experimentally characterized co-orthologs and were downloaded from the SwissProt protein database (pax2.1, PAX2_BRARE; pax2.2, O93370; sox1a, Q4V997; sox1b, Q2Z1R2). The remaining novel zebrafish orthologs were downloaded using Ensembl Compara. In the cases of $B C L 11 A$, FIGN and ZNF5O3 only a single ortholog was identified by Ensembl. In the case of $E B F 1$, no zebrafish ortholog could be identified, and was, therefore, replaced by a single ortholog from the stickleback genome. The closest available invertebrate ortholog of each gene in either Ciona (ci), Drosophila (dm) or Caenorhabditis elegans (ce) was used as an outgroup (BCL11A, LD11946p (dm); EBF1, coe (ci); FIGN, CBG21866 (ce); PAX2, pax258 (ci); SOX1, soxNRA (dm); UNC4.1, unc4 (ci); and ZNF503, noc (dm)).

The closest paralog from human, mouse, rat, dog, chicken and Fugu in each case was also included as an outgroup in each alignment (that is, BCL11A:BCL11B, FIGN:FIGN1L, PAX2:PAX5, SOX1:SOX $\left.3, E B F_{1}: E B F_{3}, Z_{2 N 5} O_{3}: Z N F 703\right)$. The closest related paralog was defined as the highest significantly scoring non-ortholog high scoring pair (HSP) in a BLASTp search of the human protein sequence against the 
SwissProt/trEMBL nr protein database. No closely related paralog could be identified for UNC4.1. A phylogenetic tree was created from each alignment using the neighbor joining (NJ) method and 1,00o bootstrap replicates using MEGA v3.1 [72].

\section{Fugu co-ortholog gene nomenclature}

F. rubripes is not a good experimental model organism due to difficulties in captive breeding and experimental manipulation. Consequently, few of its genes have been experimentally validated. Most gene predictions in the Fugu genome therefore remain novel, uncharacterized and have no current gene name. For the purposes of this study we decided upon a naming scheme for the Fugu co-orthologs that uses the same name as the human ortholog (for example, $S O X 1=\operatorname{sox} 1$ ) together with a number that denotes the specific co-ortholog (for example, sox1.1/sox1.2). This naming convention is similar to that used in early studies of zebrafish co-orthologs (for example, pax2.1 [63]) but which has now been superseded by a naming convention using letters (for example, pax2a) (ZFIN gene nomenclature guidelines [73]). We therefore used a number-based nomenclature to distinguish Fugu coorthologs from zebrafish co-orthologs. For those genes in which zebrafish co-orthologs had previously been characterized (pax2a/pax2b, sox1a/sox1b) we named Fugu equivalents by their phylogenetic similarity to these characterized zebrafish genes as ascertained through phylogeny. So, as an example, PAX2 co-orthologs were identified on Fugu scaffolds 86 and 59 (assembly v4; Figure 1d). The phylogeny identified the protein encoded on $\mathrm{S} 86$ as closest to zebrafish pax2a and that encoded on $\mathrm{S} 59$ as closest to pax2b (Figure 1c); therefore, the gene on S86 was named pax2.1 and the gene on S59 was named pax2.2. The rest of the co-orthologous sets that did not have characterized zebrafish equivalents were assigned names randomly. It is important to note this nomenclature is used purely to distinguish the genes and has no functional significance.

\section{Identification of CNEs in Fugu co-orthologous regions}

$\mathrm{CNE}$ clusters derived from the whole-genome alignment were used to define the extent of sequence in both human and Fugu for use in more sensitive multiple alignments. Regions up to the next known gene from the most peripheral CNEs in each cluster were extracted in both human and Fugu using the Ensembl API [74]. Special attention was paid to include the same orthologous region between co-orthologous pairs to ensure equivalent comparison. In situations where the full extent of the region could not be identified in one of the coorthologs due to the location of the region at the end of a scaffold (for example, scaffold_86, znf503.1; Additional data file 1), only CNEs identified up to the same orthologous region (estimated by the presence of nearby genes) in the second coortholog were used for comparative analyses. Orthologous sequences corresponding to each human region were similarly extracted in mouse (assembly v34) and rat (assembly v3.4). All genomic sequences were orientated prior to align- ment so that the trans-dev gene was in positive orientation and masked for known repeats and low complexity regions using RepeatMasker and the relevant species-specific repeat library. Multiple alignments for the discovery of conserved sub-sequences located in the same relative order and orientation were carried out using the MLAGAN alignment toolkit [75] with translated anchoring and the phylogenetic guide tree '((human (mouse rat)) fugu)'. Pairwise glocal alignments to uncover conserved elements that may have undergone rearrangements (and are, therefore, no longer in the same relative order along the sequence) or inversions between Fugu and all other organisms utilised in the MLAGAN alignment were carried out in a pairwise fashion using Shuffle-LAGAN [76] with default parameters. Each pair of Fugu co-orthologous regions was aligned to the same orthologous mammalian sequence. CNEs were identified from the alignments using the VISTA program [77] as regions with at least $65 \%$ identity over $40 \mathrm{bp}$ using Fugu as the baseline sequence. CNEs were filtered further to include only those that were conserved in human and at least one rodent.

\section{Identification of overlapping and distinct CNEs between Fugu co-orthologous regions}

The human sequence was used as a reference in order to ascertain whether CNEs identified from each co-ortholog region overlapped the same human sequence (termed 'overlapping') or were conserved in only one co-ortholog (termed 'distinct'). CNEs between co-orthologs were considered overlapping if the conserved sequence overlapped the same position in the human genome by at least $20 \mathrm{bp}$. Fugu CNEs that were defined as 'distinct' to one co-ortholog were used as query sequences against the alternative Fugu co-orthologous genomic region using the CHAOS local aligner [75] on both strands with the following parameters: word length 10, score cut-off 10, degeneracy tolerance 1 , rescoring cut-off 1,000 , and BLAST-like extension on. Resulting alignments were filtered to retain only those with at least $65 \%$ identity over 40 bp.

\section{Evolution of overlapping CNEs \\ Element length}

To ensure equivalent comparison, the length of the human $\mathrm{CNE}$ was used when measuring changes in element length between CNEs conserved in both co-orthologs. For each pair of overlapping CNEs with a one-to-one relationship (that is, each CNE overlapped one other $\mathrm{CNE}$ ), the proportion $(P)$ of the length of the overlap compared to the full length of each CNE was calculated using:

$$
P=o v / l e n
$$

where $o v$ is the length of the overlap between co-orthologous $\mathrm{CNE}$ (in bp) and len is the full length of the CNE. Values of $P$ that tend towards 1 indicate all or the majority of the element is contained within the overlap while those tending towards o 
indicate only a small proportion of the element is contained within the overlapping region.

\section{Sequence evolution}

To compare the evolutionary rates of Fugu co-orthologous copies against the single human copy (representing the ancestral sequence) we used all 'overlapping' co-orthologous CNEs. For those CNEs that did not have a one-to-one relationship (for example, in cases where two or more CNEs in one region overlapped a single CNE in another) we treated each individual overlap region independently. Multiple alignments were created for each co-ortholog CNE individually together with orthologous sequence from human, mouse, rat, dog and chicken (where available) to produce the best mapping of orthologous bases. The human sequence from the overlap detected was used to extract corresponding sequence within each multiple alignment for each co-orthologous Fugu copy together with those of orthologous sequences from the other vertebrates. These sequences were then realigned together using DIALIGN (v2.2) [78] and all gapped positions removed using the Gblocks program (vo.91b) [79]. A Tajima relative rate test of each pair of Fugu co-orthologous copies against the single human sequence was carried out as described in [57]. Only sequences that showed at least 4 independent changes in one of the elements and a $p$ value $\leq 0.05$ were considered to have undergone significant change.

\section{Identification of CNEs duplicated at the origin of vertebrates}

All human CNE sequences were searched against the human genome using BLAST with sensitive parameters (word size 8, mismatch penalty -1 ) to identify CNEs that have more than a single match (e-value $\leq 1 \times 10^{-4}$ ) in the human genome. Paralogs were identified within $1.5 \mathrm{Mb}$ of each hit using the method set out in [29]. The probability of the enrichment for overlapping CNEs within the dCNE set was calculated using a $\chi^{2}$ test with expected numbers for each type of CNE (overlapping versus distinct) calculated from the proportion of each within the whole CNE dataset (381:430 $=0.469$ :0.531).

\section{Additional data files}

The following additional data are available with the online version of this paper. Additional data file 1 is a comparison of the CNEs and genic environment between Fugu co-orthologs of znf503.1 and znf503.2. Additional data file 2 is a bar chart showing that changes in the number of CNEs between coorthologs correlates with changes in the size of the genomic region in which they are identified. Additional data file 3 is a full table of results of the relative rate tests for all overlapping co-orthologous CNEs.

\section{Acknowledgements}

We would like to thank Debbie Goode, Gayle McEwen and Wally Gilks for useful discussions during the writing of this manuscript, Lucinda Fell for help in formatting the figures and Remo Sanges for advice and use of his CHAOS parser. This work was funded by the Medical Research Council.

\section{References}

I. Lynch M, Conery JS: The evolutionary fate and consequences of duplicate genes. Science 2000, 290: I I5I-II 55.

2. Ohno S: Evolution by Gene Duplication Heidelberg: Springer-Verlag; 1970.

3. Nowak MA, Boerlijst MC, Cooke J, Smith JM: Evolution of genetic redundancy. Nature 1997, 388:167-171.

4. Nadeau JH, Sankoff D: Comparable rates of gene loss and functional divergence after genome duplications early in vertebrate evolution. Genetics 1997, 147: 1 259-1266.

5. Amores A, Force A, Yan YL, Joly L, Amemiya C, Fritz A, Ho RK, Langeland J, Prince V, Wang YL, et al.: Zebrafish hox clusters and vertebrate genome evolution. Science 1998, 282:|7|I-|7|4.

6. Hughes MK, Hughes AL: Evolution of duplicate genes in a tetraploid animal, Xenopus laevis. Mol Biol Evol 1993, 10:1360-1369.

7. Force A, Lynch M, Pickett FB, Amores A, Yan YL, Postlethwait J: Preservation of duplicate genes by complementary, degenerative mutations. Genetics 1999, I 5 I: 153 |-1545.

8. Istrail S, Davidson EH: Logic functions of the genomic cis-regulatory code. Proc Natl Acad Sci USA 2005, 102:4954-4959.

9. Howard ML, Davidson EH: Cis-regulatory control circuits in development. Dev Biol 2004, 27 I: 109-II8.

10. Yuh $\mathrm{CH}$, Bolouri $\mathrm{H}$, Davidson $\mathrm{EH}$ : Cis-regulatory logic in the endo 16 gene: switching from a specification to a differentiation mode of control. Development 200I, 128:617-629.

II. Carroll SB: Evolution at two levels: on genes and form. PLoS Biol 2005, 3:e245.

12. Postlethwait JH, Yan YL, Gates MA, Horne S, Amores A, Brownlie A, Donovan A, Egan ES, Force A, Gong Z, et al.: Vertebrate genome evolution and the zebrafish gene map. Nat Genet 1998, I 8:345-349.

13. Taylor JS, Van de Peer Y, Braasch I, Meyer A: Comparative genomics provides evidence for an ancient genome duplication event in fish. Philos Trans R Soc Lond B Biol Sci 200I, 356:166I-1679.

14. Taylor JS, Braasch I, Frickey T, Meyer A, Van de Peer Y: Genome duplication, a trait shared by 22,000 species of ray-finned fish. Genome Res 2003, 13:382-390.

15. Christoffels A, Koh EG, Chia JM, Brenner S, Aparicio S, Venkatesh B: Fugu genome analysis provides evidence for a whole-genome duplication early during the evolution of ray-finned fishes. Mol Biol Evol 2004, 2 I: I | 46-I I II.

16. Jaillon O, Aury JM, Brunet F, Petit JL, Stange-Thomann N, Mauceli E, Bouneau L, Fischer C, et al:: Genome duplication in the teleost fish Tetraodon nigroviridis reveals the early vertebrate protokaryotype. Nature 2004, 43 I:946-957.

17. Vandepoele K, De Vos W, Taylor JS, Meyer A, Van de Peer Y: Major events in the genome evolution of vertebrates: paranome age and size differ considerably between ray-finned fishes and land vertebrates. Proc Natl Acad Sci USA 2004, I0 I: 1638-1643.

18. Cresko WA, Yan YL, Baltrus DA, Amores A, Singer A, RodriguezMari A, Postlethwait JH: Genome duplication, subfunction partitioning, and lineage divergence: Sox 9 in stickleback and zebrafish. Dev Dyn 2003, 228:480-489.

19. Yu WP, Brenner S, Venkatesh B: Duplication, degeneration and subfunctionalization of the nested synapsin-Timp genes in Fugu. Trends Genet 2003, 19:180-183.

20. Scholpp S, Brand M: Morpholino-induced knockdown of zebrafish engrailed genes eng2 and eng3 reveals redundant and unique functions in midbrain - hindbrain boundary development. Genesis 200I, 30:129-133.

21. Flores MV, Tsang VW, Hu W, Kalev-Zylinska M, Postlethwait J, Crosier P, Crosier K, Fisher S: Duplicate zebrafish runx2 orthologs are expressed in developing skeletal elements. Gene Expr Patterns 2004, 4:573-58I.

22. Santini S, Boore JL, Meyer A: Evolutionary conservation of regulatory elements in vertebrate Hox gene clusters. Genome Res 2003, I3:IIII-II22.

23. McClintock JM, Kheirbek MA, Prince VE: Knockdown of duplicated zebrafish hoxb I genes reveals distinct roles in hindbrain patterning and a novel mechanism of duplicate gene retention. Development 2002, 129:2339-2354.

24. Postlethwait J, Amores A, Cresko W, Singer A, Yan YL: Subfunction partitioning, the teleost radiation and the annotation of the human genome. Trends Genet 2004, 20:48I-490.

25. Boffelli D, Nobrega MA, Rubin EM: Comparative genomics at the vertebrate extremes. Nat Rev Genet 2004, 5:456-465.

26. Woolfe A, Goodson M, Goode DK, Snell P, McEwen GK, Vavouri T, 
Smith SF, North P, Callaway H, Kelly K, et al:: Highly conserved non-coding sequences are associated with vertebrate development. PLoS Biol 2005, 3:e7.

27. Sandelin A, Bailey P, Bruce S, Engstrom PG, Klos JM, Wasserman WW, Ericson J, Lenhard B: Arrays of ultraconserved non-coding regions span the loci of key developmental genes in vertebrate genomes. BMC Genomics 2004, 5:99.

28. Bejerano G, Pheasant M, Makunin I, Stephen S, Kent WJ, Mattick JS, Haussler D: Ultraconserved elements in the human genome. Science 2004, 304: I32I-I325.

29. McEwen GK, Woolfe A, Goode D, Vavouri T, Callaway H, Elgar G: Ancient duplicated conserved noncoding elements in vertebrates: a genomic and functional analysis. Genome Res 2006, 16:45I-465.

30. Ovcharenko I, Loots GG, Nobrega MA, Hardison RC, Miller W, Stubbs $L$ : Evolution and functional classification of vertebrate gene deserts. Genome Res 2005, 15:|37-145.

31. Ahitur N, Prabhakar S, Poulin F, Rubin EM, Couronne O: Mapping cis-regulatory domains in the human genome using multispecies conservation of synteny. Hum Mol Genet 2005, 14:3057-3063.

32. Nobrega MA, Ovcharenko I, Afzal V, Rubin EM: Scanning human gene deserts for long-range enhancers. Science 2003, 302:4I3.

33. de la Calle-Mustienes E, Feijoo CG, Manzanares M, Tena JJ, Rodriguez-Seguel E, Letizia A, Allende ML, Gomez-Skarmeta JL: A functional survey of the enhancer activity of conserved noncoding sequences from vertebrate Iroquois cluster gene deserts. Genome Res 2005, I 5:106|-1072.

34. Goode DK, Snell P, Smith SF, Cooke JE, Elgar G: Highly conserved regulatory elements around the SHH gene may contribute to the maintenance of conserved synteny across human chromosome 7q36.3. Genomics 2005, 86: 172-181.

35. Shin JT, Priest JR, Ovcharenko I, Ronco A, Moore RK, Burns CG, MacRae CA: Human-zebrafish non-coding conserved elements act in vivo to regulate transcription. Nucleic Acids Res 2005, 33:5437-5445.

36. Pennacchio LA, Ahituv N, Moses AM, Prabhakar S, Nobrega MA Shoukry M, Minovitsky S, Dubchak I, Holt A, Lewis KD, et al:: In vivo enhancer analysis of human conserved non-coding sequences. Nature 2006, 444:499-502.

37. Vavouri T, Walter K, Gilks WR, Lehner B, Elgar G: Parallel evolution of conserved non-coding elements that target a common set of developmental regulatory genes from worms to humans. Genome Biology 2007 in press.

38. Roest Croullius H, Weissenbach J: Fish genomics and biology. Genome Res 2005, 15:1675-1682

39. Van de Peer Y, Taylor JS, Joseph J, Meyer A: Wanda: a database of duplicated fish genes. Nucleic Acids Res 2002, 30: 109-I I 2.

40. Burton $\mathrm{Q}$, Cole LK, Mulheisen M, Chang W, Wu DK: The role of Pax2 in mouse inner ear development. Dev Biol 2004 272:16I-I75.

41. Dressler GR, Deutsch U, Chowdhury K, Nornes HO, Gruss P: Pax2, a new murine paired-box-containing gene and its expression in the developing excretory system. Development 109:787-795.

42. Nornes HO, Dressler GR, Knapik EW, Deutsch U, Gruss P: Spatially and temporally restricted expression of Pax2 during murine neurogenesis. Development 1990, 109:797-809.

43. Malas S, Duthie SM, Mohri F, Lovell-Badge R, Episkopou V: Cloning and mapping of the human SOXI: a highly conserved gene expressed in the developing brain. Mamm Genome 1997, 8:866-868.

44. Pevny LH, Sockanathan S, Placzek M, Lovell-Badge R: A role for SOXI in neural determination. Development 1998 , I 25:1967-1978

45. Saiki $Y$, Yamazaki Y, Yoshida M, Katoh O, Nakamura T: Human EVI9, a homologue of the mouse myeloid leukemia gene, is expressed in the hematopoietic progenitors and down-regulated during myeloid differentiation of HL60 cells. Genomics 2000, 70:387-391.

46. Gisler R, Jacobsen SE, Sigvardsson M: Cloning of human early Bcell factor and identification of target genes suggest a conserved role in B-cell development in man and mouse. Blood 2000, 96: 1457-| 464 .

47. Akerblad P, Lind U, Liberg D, Bamberg K, Sigvardsson M: Early Bcell factor $(O / E-I)$ is a promoter of adipogenesis and involved in control of genes important for terminal adipocyte differentiation. Mol Cell Biol 2002, 22:80I5-8025.

48. Cox GA, Mahaffey CL, Nystuen A, Letts VA, Frankel WN: The mouse fidgetin gene defines a new role for AAA family proteins in mammalian development. Nat Genet 2000, 26:198-202.

49. Rovescalli AC, Asoh S, Nirenberg M: Cloning and characterization of four murine homeobox genes. Proc Natl Acad Sci USA 1996, 93:10691-10696.

50. Mansouri A, Yokota Y, Wehr R, Copeland NG, Jenkins NA, Gruss P: Paired-related murine homeobox gene expressed in the developing sclerotome, kidney, and nervous system. Dev Dyn 1997, 210:53-65.

5I. Chang CW, Tsai CW, Wang HF, Tsai HC, Chen HY, Tsai TF, Takahashi H, Li HY, Fann MJ, Yang CW, et al.: Identification of a developmentally regulated striatum-enriched zinc-finger gene, Nolz-I, in the mammalian brain. Proc Natl Acad Sci USA 2004 101:2613-2618.

52. Steinke D, Salzburger W, Braasch I, Meyer A: Many genes in fish have species-specific asymmetric rates of molecular evolution. BMC Genomics 2006, 7:20-38

53. The CONDOR Database [http://condor.fugu.biology.qmul.ac.uk]

54. McLysaght A, Enright AJ, Skrabanek L, Wolfe KH: Estimation of synteny conservation and genome compaction between pufferfish (Fugu) and human. Yeast 2000, 17:22-36.

55. Aparicio S, Chapman J, Stupka E, Putnam N, Chia JM, Dehal P, Christoffels A, Rash S, Hoon S, Smit A, et al:: Whole-genome shotgun assembly and analysis of the genome of Fugu rubripes. Science 2002, 297:130I-I310.

56. Mackenzie A, Miller KA, Collinson JM: Is there a functional link between gene interdigitation and multi-species conservation of synteny blocks? Bioessays 2004, 26: 12 I7-I224.

57. Tajima F: Simple methods for testing the molecular evolutionary clock hypothesis. Genetics 1993, I 35:599-607.

58. Dehal $\mathrm{P}$, Boore $\mathrm{LL}$ : Two rounds of whole genome duplication in the ancestral vertebrate. PLOS Biol 2005, 3:e3/4.

59. Vavouri T, McEwen GK, Woolfe A, Gilks WR, Elgar G: Defining a genomic radius for long-range enhancer action: duplicated conserved non-coding elements hold the key. Trends Genet 2006, 22:5-10.

60. Lynch M, Force A: The probability of duplicate gene preservation by subfunctionalization. Genetics 2000, 154:459-473.

6I. McBride DJ, Kleinjan DA: Rounding up active cis-elements in the triple $C$ corral: combining conservation, cleavage and conformation capture for the analysis of regulatory gene domains. Brief Funct Genomic Proteomic 2004, 3:267-279.

62. Tumpel S, Cambronero F, Wiedemann LM, Krumlauf R: Evolution of cis elements in the differential expression of two Hoxa2 coparalogous genes in pufferfish (Takifugu rubripes). Proc Natl Acad Sci USA 2006, 103:5419-5424.

63. Pfeffer PL, Gerster T, Lun K, Brand M, Busslinger M: Characterization of three novel members of the zebrafish Pax2/5/8 family: dependency of Pax5 and Pax8 expression on the Pax2 I (noi) function. Development 1998, I25:3063-3074.

64. Okuda $\mathrm{Y}$, Yoda H, Uchikawa M, Furutani-Seiki M, Takeda H, Kondoh $\mathrm{H}$, Kamachi $\mathrm{Y}$ : Comparative genomic and expression analysis of group BI sox genes in zebrafish indicates their diversification during vertebrate evolution. Dev Dyn 2006 235:8II-825

65. Aburomia $R$, Khaner $O$, Sidow $A$ : Functional evolution in the ancestral lineage of vertebrates or when genomic complexity was wagging its morphological tail. J Struct Funct Genomics 2003, 3:45-52

66. Durand D: Vertebrate evolution: doubling ad shuffling with a full deck. Trends Genet 2003, 19:2-5.

67. Nelson JS: Fishes of the World New York: John Wiley and Sons; 1994

68. Hoegg S, Brinkmann H, Taylor JS, Meyer A: Phylogenetic timing of the fish-specific genome duplication correlates with the diversification of teleost fish. I Mol Evol 2004, 59: 190-203.

69. Volff JN: Genome evolution and biodiversity in teleost fish Heredity 2005, 94:280-294.

70. Harris MA, Clark J, Ireland A, Lomax J, Ashburner M, Foulger R, Eilbeck K, Lewis S, Marshall B, Mungall C, et al.: The Gene Ontology (GO) database and informatics resource. Nucleic Acids Res 2004, 32:D258-26I.

7I. Thompson JD, Higgins DG, Gibson T]: CLUSTAL W: improving the sensitivity of progressive multiple sequence alignment through sequence weighting, position specific gap penalties and weight matrix choice. Nucleic Acids Res 1994, 22:4673-4680.

72. Kumar S, Tamura K, Nei M: MEGA3: Integrated software for Molecular Evolutionary Genetics Analysis and sequence alignment. Brief Bioinform 2004, 5:150-163. 
73. Zebrafish Nomenclature Guidelines [http://zfin.org/zf_info/ nomen.html]

74. Birney E, Andrews D, Caccamo M, Chen Y, Clarke L, Coates G, Cox T, Cunningham F, Curwen V, Cutts T, et al.: Ensembl 2006. Nucleic Acids Res 2006, 34:D556-561.

75. Brudno M, Chapman M, Gottgens B, Batzoglou S, Morgenstern B: Fast and sensitive multiple alignment of large genomic sequences. BMC Bioinformatics 2003, 4:66-77.

76. Brudno M, Malde S, Poliakov A, Do CB, Couronne O, Dubchak I, Batzoglou S: Glocal alignment: finding rearrangements during alignment. Bioinformatics 2003, 19:i54-62.

77. Mayor C, Brudno M, Schwartz JR, Poliakov A, Rubin EM, Frazer KA, Pachter LS, Dubchak I: VISTA: visualizing global DNA sequence alignments of arbitrary length. Bioinformatics 2000, 16:1046-1047.

78. Morgenstern B: DIALIGN: multiple DNA and protein sequence alignment at BiBiServ. Nucleic Acids Res 2004, 32:W33-36.

79. Castresana J: Selection of conserved blocks from multiple alignments for their use in phylogenetic analysis. Mol Biol Evol 2000, I 7:540-552. 